\title{
Helminth Parasites of Pigs and Development of Suitable Strategy for Its Control
}

\author{
N. Baskota*1 and S. P. Shrestha ${ }^{2}$ \\ ${ }^{1}$ Swine and Avian Research Program, Khumaltar \\ ${ }^{2}$ Animal Health Research Division, NARC \\ *Corresponding author: neerajbaskota@gmail.com
}

\begin{abstract}
The study aimed to formulate the suitable development strategies to control helminthiasis in pig by understanding the nature of the epidemiological pattern that is yet to exploit to further enhance its productivity. A longitudinal study was done to find the general prevalence pattern of helminth parasites from June/ July 2017 to May/June 2018 in the research farm of Swine and Avian Research Program, Khumaltar. A total of 50 animals of different age groups were considered for the study and they were deprived of antihelminthic treatment throughout the study period. The fecal samples were collected at 3-4 months interval and EPG was done as per the standard protocol. Three different groups of antihelminthic were used including a control group as different treatments and they were assigned as T1-Pyrantel pamoate, T2-Benzimidazoles, T-3 Imidazothiazole, and T-4 Control. The EPG was done for at least 3-week post-treatment. The result of this study showed the significant effect of the season $(p<0.05)$ as a high prevalence of roundworms especially Ascaris (60\%) and Strongylus (40\%) during June/ July with more than 30 cases. However, a slight increase in parasitic prevalence was seen in September/October and $\mathrm{Jan} / \mathrm{Feb}$ too. Similarly, all the antihelminthics used were 100\% effective ( $>0.05)$ against these roundworms. The female and young groups of animals showed remarkable symptoms of parasitic infections compared to the male ones. In conclusion, the different groups of drugs demonstrated very satisfactory performances in terms of the removal of parasites for good health of pigs and these should be given at 3-4 months interval as routine deworming schedule.
\end{abstract}

Keywords: Antihelminthics, Helminth parasites, Pig, Productivity

\section{INTRODUCTION}

Pig and pork productions are an important component of the subsistence agriculture system in Nepal and are raised by different communities of Nepalese society for their diverse use. The population of pig has increased gradually from 0.76 million to 1.2 million whereas pork production has increased from 0.13 metric tons to 0.24 metric tons in 15 years of period (FAO, 2014). The increasing trend in the number indicates that pig and pork have played an important role in Nepalese agriculture for many years. Simultaneously, many constraints like the trend of parasites have been increasing as well. Loss of piglets during the parturition process and the pre-weaning period is one of the 
serious causes of the reduced production efficiency in the pig breeding herd due to diseases caused by different helminth parasites (Zanga et. al., 2003).

The major diseases affecting pig production are internal helminth parasites (Zanga et.al., 2003; Mul, 2007). Although endo parasitism has been frequently reported, limited study has been carried out in the country. The role of parasitism is important in village reared pigs throughout the territory and contributes substantially to high-mortality and retarded growth. There is still a lack of wellmanaged study on such a sensitive issue in the country. The exact loss due to these causes is yet to exploit. Swine endoparasitism is heterogeneous regarding the species involved and pathogenicity of the parasites (Roepstorff et.al., 1998; Urquhart et.al., 1996).

The effects of endoparasites depend on the parasite load and the individual resistance of the host, which are greatly influenced by environmental and nutritional factors (Banerjee, 1980). Endoparasitism can occur with or without the presence of clinical symptoms. In the first case, it can be lethal, especially in the initial growth phase. The absence of clinical symptoms is important for production because if it goes unnoticed, it can cause economic loss due to the reduction in pig performance (Saeed and Roeperstoff, 2001). Endoparasitism does not manifest itself as a relevant problem in current pig farming. This is due to new technology, and above all, those technologies related to the interruption of the parasitic cycles, such as confinement facilities when used with specific prophylactic techniques. However, not all preventive techniques can be practiced in outdoor free-range or deep litter systems.

Parasites act as a stressor stimulus to animal metabolism, altering the nutritional demands of the host (Petkevicius et. al., 1999). However, little is known about the implications of the interaction of endoparasitism with nutritional components and animal performance. Given, the study aimed to formulate the suitable development strategies by understanding the nature of the epidemiological pattern that is yet to exploit and enhance productivity.

\section{MATERIALS AND METHODS}

\section{Site Selection}

The experiment was conducted in the Farm of Swine and Avian Research Program, Khumaltar, Lalitpur District located in the Central Development Region of the country. The geographical diversity ranges from 1400 masl.

\section{Study on Management Practices of Pig Production.}

The pigs managed in Swine and Avian Research Program, Khumaltar were provided 2-4 kgs of greens and plenty of fresh water per day and vaccination against swine fever, Foot and Mouth Disease was done as per routine. The female pigs attending heat were made to conceive with pure breeds of its own, however avoiding inbreeding. The pregnant and gestated pigs were handled as per recommendations. The general practices of hygiene and bio-security maintenance were followed. 


\section{Epidemiological Sstudy on Parasitic Occurrence}

The routine farm visit, fecal collection from each pig and piglets were done (two/three month's interval) with suitable labeling in zip lock bag and brought in the laboratory of Animal Health Research Division, Khumaltar. Egg per gram described by (Soulsby et.al., 2008) was carried out to explore general prevalence.

\section{RESULT AND DISCUSSION}

The epidemiological pattern of the parasitic infection obtained revealed the following results which have been given through Figure 1 enlisted below

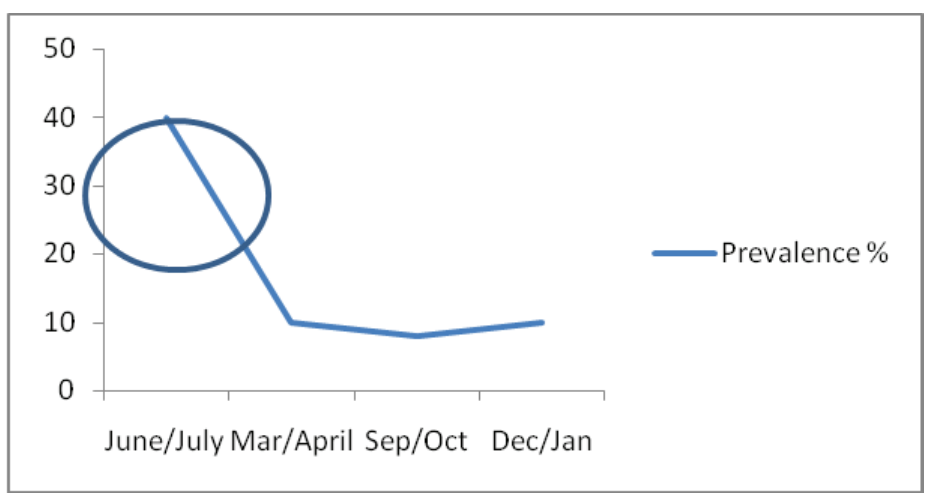

Figure 1: Parasitic occurrence in different months.

The above figure indicate that the parasitic incidence was higher in June/July representing $40 \%$ (40 out of 100) of prevalence. These patterns were found to decrease in the remaining months ranging from 10-15\% (15 positive cases) in March/April, August/September. The difference in temperature, relative humidity, and the rainfall pattern might be associated with the outcome. The temperature and rainfall of the June/ July ranged between $28-32^{\circ} \mathrm{c}$ and $1000 \mathrm{~mm}$ while the same of the remaining months were less. This result resembles that of Martin et.al., 1988 advocating about warm and wet conditions providing a very suitable environment for the survival and multiplications of insects/parasites as a vector of disease. In this study, the month of June/July revealed the warmest and wet season compared to others. 
Types of Parasites : The Ascaris suum, Strongylus, and mixed infection were the principle parasites as per the findings. Both Ascaris Suum and Strongylus were highest in June/July and their incidence decreased in the remaining months. No other species were reported (Figure 2).

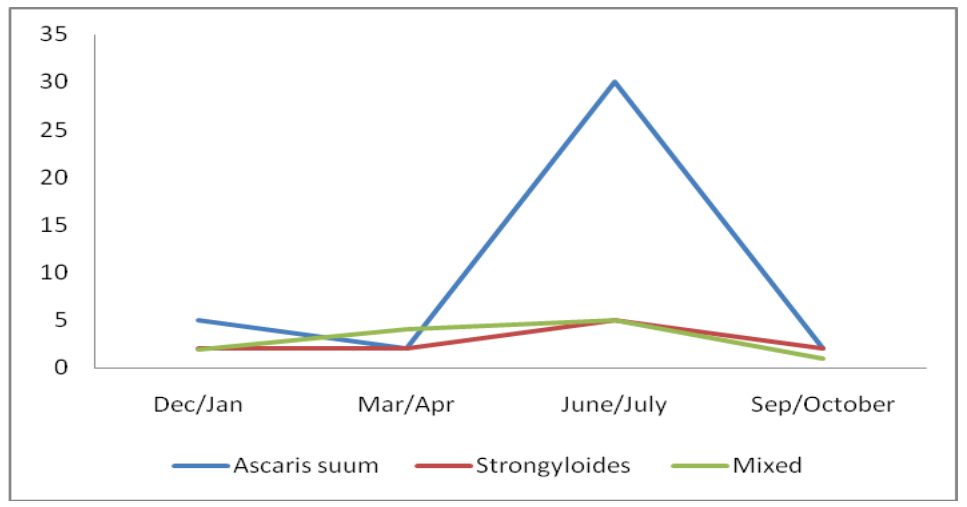

Figure 2: Different types of parasites infecting the animals have been presented by the graph

Thus, these findings contradict that of Thakur, 1998 reporting more than six to seven species in the eastern hills of Nepal. Similarly, in the study carried out by Tablot, 1970 in Papua New Guinea showed more than 25 different types of parasites. The difference in geography, management pattern and the sample size might be the causes of such variation.

\section{Age-wise Prevalence Pattern}

In the different groups of piglets, animals below 4 months showed a high prevalence of Ascaris and the animals above 9 months showed a prevalence of both Ascaris and Strongylous. However, there was a mixed infection for the animals between 4-9 months of age. Our results were similar to the findings by Thakur, 1997 and stated that the age of pigs has an effect on the level of infection especially for Ascaris, unlike others. The exact reason could not be explored, though it is thought to be due to the development of age-related immunity in piglets.

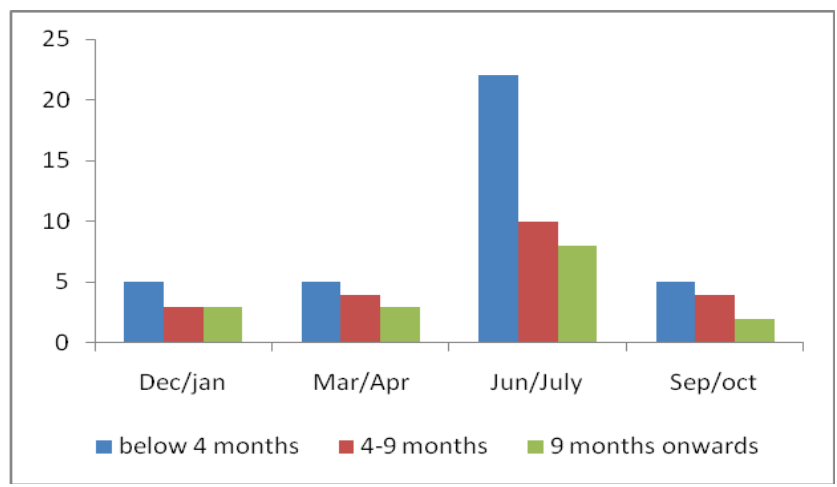

Figure 3: Age-wise prevalence pattern of the parasites 


\section{Conduction of Trial Using Several Groups of Antihelminthics}

Different groups of antihelminthics available in the market were used for the conduction of trial at their recommended doses. Pyrantel pamoate was provided to the younger ones from one week to 2 months of age and the rest of the animals aging one month onwards was provided with benzimidazoles, imidazothiazoles, and control group. The initial and later EPG count was done according to the standard protocol provided by (Soulsby et. al., 2008). Egg reduction count was done at the interval of one week until three weeks and the data were recorded as well. The result is given below.

Table 1: Group of drugs and their effectiveness

\begin{tabular}{lcccc}
\hline Group of Drugs & Initial Count & $1^{\text {st }}$ week & $2^{\text {nd }}$ week & $3^{\text {rd }}$ week \\
\hline T1- Pyrantel pamoate & 15 & 2 & 1 & 0 \\
T2- Benzimidazoles & 16 & 1 & 0 & 0 \\
T-3 Imidazothiazoles & 15 & 1 & 0 & 0 \\
T-4 Control & 15 & 18 & 20 & 22 \\
\hline
\end{tabular}

Results obtained from the trial revealed a significant effect of treatment over the control. All the groups of drugs were $100 \%$ effective against the helminth parasites that existed till 28 days postexperiment.

Table 2: Average egg count per gram from the samples of trial goats during the pre- and posttreatment periods

\begin{tabular}{ccccccccccc}
\hline Day & $\begin{array}{c}\mathrm{T}_{1} \\
\text { (mean) }\end{array}$ & $\begin{array}{c}\text { Mean \% } \\
\text { reduction }\end{array}$ & $\begin{array}{c}\mathrm{T}_{2} \\
\text { (mean) }\end{array}$ & $\begin{array}{c}\text { Mean \% } \\
\text { reduction }\end{array}$ & $\begin{array}{c}\mathrm{T} 3 \\
\text { (mean) }\end{array}$ & $\begin{array}{c}\text { Mean } \% \\
\text { reduction }\end{array}$ & $\begin{array}{c}\mathrm{T}_{3} \\
\text { (mean) }\end{array}$ & $\begin{array}{c}\text { Mean \% } \\
\text { increased }\end{array}$ & $\begin{array}{c}\text { P- } \\
\text { value }\end{array}$ & $\begin{array}{c}\text { Level of } \\
\text { significance }\end{array}$ \\
\hline 0 & 584.5 & 0 & 436.2 & 0 & 436.2 & 0 & 281.6 & 0 & 0.04 & $* * *$ \\
7 & 8.7 & 98.5 & 24.2 & 94.5 & 24.2 & 94.5 & 296.6 & 5.4 & 0.02 & $* * *$ \\
14 & 0 & 100 & 12.0 & 97.3 & 12.0 & 97.3 & 455.9 & 61.95 & 0.00 & $* * *$ \\
21 & 0 & 100 & 8.7 & 98.0 & 8.7 & 98.0 & 586.4 & 108.2 & 0.00 & $* * *$ \\
28 & 0 & 100 & 5.3 & 98.8 & 5.3 & 98.8 & 755.9 & 168.5 & 0.00 & $* * *$ \\
\hline
\end{tabular}

Note $^{* * *}=$ Level of significance at $0.01 \%$

The finding is under that of different researchers. The efficacy of ivermectin against both ectoand endoparasites in the present study corresponded to the findings of earlier studies by Ratnaparhki et.al. 1996; Mahbub et.al., 1992. The studies reported 89.5\%-100\% efficacy against endo- and ectoparasitic infection in goats or other small ruminants reared in a backyard system in Bangladesh and overseas. A slight variation of the efficacy of ivermectin in those previous studies might be due to the degree of ectoparasitic infection, the sample size used for different experiments, dose and preparation, and the route used for administering the drugs. However, ivermectin proved its efficacy in the present study without any adverse effect. 


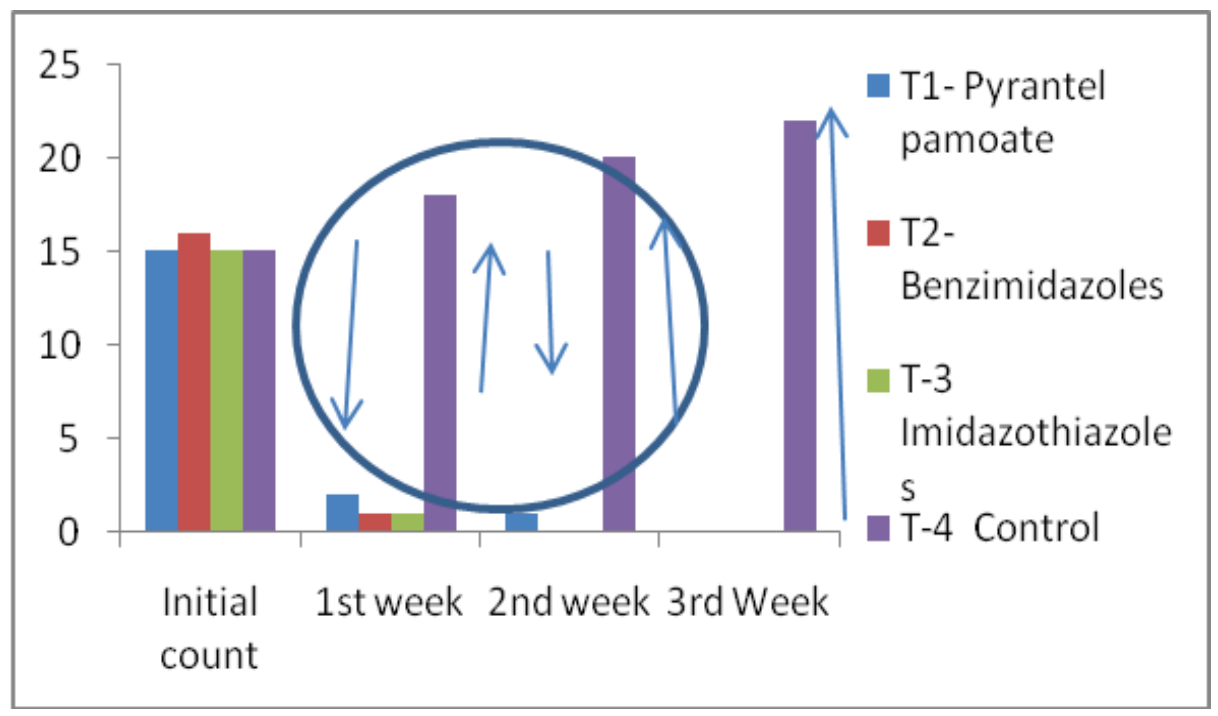

Figure 4: Result of the trial using several groups of anthelminthics

The effective performance of triclabendazole along with levamisole against endoparasites in the current study also coincided with the performance of triclabendazole alone reported earlier in backyard goats and sheep (Mahbub, 1996 and Zanga et.al, 2003). Moreover, using the levamisole (with triclabendazole) in this study could have modulated the body's immune system to give necessary protection against different infectious organisms. However, this study has not studied immune status with the administration of levamisole.

\section{CONCLUSION}

The present study revealed that the helminth parasite is the major cause of helminthiasis in pig in Nepal which affects the pig productivity and further shows the detrimental effect on the pig industry. This study was conducted to formulate different strategies to control helminthiasis in pig and from this conducted research, the prevalence pattern of parasites was found to be high in June/July (Rainy season). Similarly, different groups of antihelminthics used in this trial were found to be $100 \%$ effective. However, due to the sporadic occurrence of parasites throughout the year, deworming should be done at the interval of 3-4 months interval.

\section{ACKNOWLEDGMENT}

I express many thanks and gratitude to Mr. Damodar Neupane (Co-ordinator of Swine and Avian Research Program) for his proficient supervision, advice and constructive criticism that facilitated to create this work. I am also indebted to Dr.Swoyam Prakash Shrestha (ChiefAHRD), Khumaltar for his continuous supervision regular guidance, data analysis and all the efforts he made to bring this manuscript in present form. My appreciation also goes to Babulal Kanwar, Mr. Rabin Chaudhary, and Mr. Basant Khatri, NARC for their regular guidance. I am grateful to all the staff of Swine and Avian Research Program, Khumaltar. 


\section{REFERENCES}

Banerjee, G.C.:1980 A Textbook of Animal Husbandry. 4th edn., Oxford and IBM Publishing Company, New Delhi. 1980.

FAO. (2014). Poultry sector in Nepal. Animal Production and Health Livestock Country Reviews. 59:29-44.

Mahbub, A.: Anthelmintic efficacy against fasciliasis and gastrointestinal nematodiasis with haemato-biochemical changes in sheep and goats. M.Sc Thesis. Bangladesh Agricultural University, Mymensingh, Bangladesh. 1996 (in Bengali).

Mahbub, A.: Anthelmintic efficacy against fasciliasis and gastrointestinal nematodiasis with haemato-biochemical changes in sheep and goats. M.Sc Thesis. Bangladesh Agricultural University, Mymensingh, Bangladesh. 1996 (in Bengali).

Mul, F.I., 2007. Intestinal parasites of Free ranging, semi captive and captive pigs in Sumatra, indonesia. Journal article, 28 (2), p.407-420.

Petkevicius S, Nansen, P., 1998, Bach Knudsen, K.E. andSkjoth, F., 1999. The effect of increasing levels of insoluble dietryfibre on the establishment and persistence of Oesophastomumdentatum in pigs, Parasite, 6, p.17-26.

Ratnaparhki, M.R., Shastri, U.V., Narlandkar, B.W., Digraskar, S.U., Degloorkar, N.M.: A note on efficacy of some flukicides against Fasciola infection in domestic animals. Indian J. Vet. Med., 1992; 12: 20.

Roperstorff, A.Jorgensen, R.J., Nansen, P. Henrisken, S. A., Skogarrad Petersen, J., and Andreasen, M., 1992. Parasiter hos okologiskeservin. Rapport over projektfinansieretafJordbrugsderektoratet under Landbrugsministeriet.Landsudvalget for Svin. Danske Slagterier, kobenhavn, Denmark, p.36

Saeed, I. andRoeperstorf F.A., 2001. Optimization of the agar -gel method for isolation of Migrating Ascaris-suum Larvae from liver and lungs of pig. Acta Veterinary Journal, scand, 42 (2). P 279-286.

Soulsby, E.J.L.: Helminths, Arthropods and Protozoa of Domesticated Animals. 7th edn., Bailliere Tindall, London. 1986.

Talbot, N.T., 1970. Incidence and distribution of helminth and arthropod parasites of indigenious owned pigs in Papua New Guinea, Department of Agriculture, stuck and Fisheries, Port Moresby, Papua New Guinea.

Thakur, R.P., 2003 Control of endoparasitic disease of pigs in the eastern hills of Nepal. Vetcon, 2003

Thakuri, K.C. 1996. Diesease of pig in the eastern hills of Nepal, Veterinery Review, 11 (2), p.33-36.

Urquhart G.M. Armour, J. Dunn, A.M.and Jennings, F.W., 1996. Veterinery Parasitology, $2^{\text {nd }}$ edition. Blackwell Science Ltd. London, p.3-140

Zanga J, Chimonyo M, Kanengoni A, Dzama K, Mukaratirwa S (2003) A comparison of the susceptibility of growing Mukota and Large White pig genotypes to inf ection with Ascaris suum. Veterinary Research Communications 27, 653-660. doi:10.1023/A:1027320428646 